\title{
ANÁLISE BIOMECÂNICA E HISTOLÓGICA DE MÚSCULOS GASTROCNÊMIOS DE RATAS SUBMETIDAS À LESÃO MUSCULAR E TRATADOS COM LASERTERAPIA DE BAIXA INTENSIDADE
}

\author{
BIOMECHANICAL AND HISTOLOGICAL ANALYSIS OF THE GASTROCNEMIUS IN RATS \\ SUBJECTED TO MUSCLE INJURY AND TREATMENT WITH LOW-LEVEL LASER THERAPY
}

Maurício José Falcai', Vanessa Vilela Monte-Raso', Rodrigo Okubo', Ariane Zamarioli', Leonardo César Carvalho', Antônio Carlos Shimano²

\section{RESUMO}

Objetivo: Avaliar, mecânica e histologicamente, a aplicação do laser terapêutico de baixa potência, no processo reparativo de lesões provocadas por impacto em músculos gastrocnêmios de ratas. Métodos: 45 ratas Wistar foram divididas em três grupos (n=15/grupo): C (controle, sem lesão), LM (lesão muscular) e LM-L (lesão muscular e laserterapia). A lesão muscular experimental foi produzida pela queda de uma carga de $250 \mathrm{~g}$, de uma altura de $30 \mathrm{~cm}$, diretamente sobre o músculo. Os animais do grupo LM-L foram submetidos à aplicação de laser 960nm, $2 \mathrm{~J} / \mathrm{cm}^{2}$, durante três dias, duas aplicações por dia, no local da lesão. Foi realizado ensaio mecânico na máquina universal de ensaio Emic ${ }^{\circledR}$. Resultados: As médias dos valores de força máxima foram: $(35,70 \pm 2,69) \mathrm{N}$ no grupo $\mathrm{C}$, $(31,77 \pm 2,59) \mathrm{N}$ no grupo LM e $(34,36 \pm 3,63) \mathrm{N}$ no grupo LM-L, com diferença estatística significativa nos grupos C e LM $(\mathrm{p}<0,05)$. As médias dos valores de rigidez relativa foram $(3,75 \pm 0,98) \mathrm{N} / \mathrm{mm}$ no grupo C, $(3,84 \pm 0,32) \mathrm{N} / \mathrm{mm}$ no grupo LM e $(4,43 \pm 0,68)$ $\mathrm{N} / \mathrm{mm}$ no grupo LM-L, sem diferença estatística ( $\mathrm{p}>0,05)$. A análise histológica evidenciou presença de vasos sanguíneos no grupo LM-L e hematomas em processo de reparação. Conclusão: A laserterapia influencia de forma positiva no processo de regeneração da lesão muscular.

Descritores - Terapia a laser de baixa intensidade; Ratos; Regeneração

\section{ABSTRACT}

Objective: To evaluate, by means of biomechanical and histological analysis, the effect of low-level laser therapy on the gastrocnemius of rats subjected to muscle lesion by impact. Methods: 45 female Wistar rats, weighing 250g, were divided into 3 groups (n=15/group): C (control), ML (animals submitted to muscle lesion) and ML-L (animals submitted to muscle lesion and laser therapy). The animals from groups $M L$ and $M L-L$ were subjected to an experimental muscle lesion by means of an impact of a $250 \mathrm{~g} \mathrm{load}$, released from a height of $30 \mathrm{~cm}$, directly on the gastrocnemius. The animals from group $M L-L$ were submitted to a treatment with a $960 \mathrm{Nm}$ laser, $2 \mathrm{~J} / \mathrm{cm}^{2}$, for 3 days, twice a day. Results: The mechanical tests were performed on the Universal Testing Machine. The average values of maximum force were: $35.70( \pm 2.69) \mathrm{N}$ in group $C$, $31.77( \pm$ 2.59) $N$ in group $M L$ and $34.36( \pm 3.63) N$ in group $M L-L$, with a statistically significant difference between groups $C$ and $M L$ $(p<0.05)$. The average values of stiffness were: $3.75( \pm 0.98)$ $\mathrm{N} / \mathrm{mm}$ in group C, $3.84( \pm 0.32) \mathrm{N} / \mathrm{mm}$ in group $M L$ and 4.43 $( \pm 0.68) \mathrm{N} / \mathrm{mm}$ in group $M L-L$, with no statistically significant differences ( $p>0.05)$. Histological analysis showed a presence of blood vessels in group $M L-L$ and a hematoma in the healing stage. Conclusion: Treatment with low-level laser had a positive effect in the healing process of muscle injury.

Keywords - Laser therapy, low-level; Rats; Regeneration

1 - Pós-Graduando do Departamento de Biomecânica, Medicina e Reabilitação do Aparelho Locomotor da Faculdade Medicina de Ribeirão Preto, USP.

2 - Professor Doutor do Departamento de Biomecânica, Medicina e Reabilitação do Aparelho Locomotor da Faculdade Medicina de Ribeirão Preto, USP.

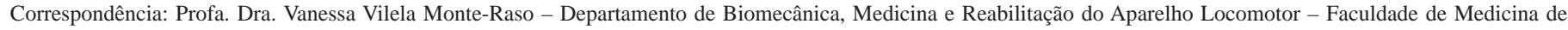
Ribeirão Preto da Universidade de São Paulo - 14048-900 - Ribeirão Preto, SP, Brasil - Campus Universitário - E-mail: vanmonteraso@yahoo.com.br 


\section{INTRODUÇÃO}

Dentre as lesões musculares, 90\% são contusões ou estiramento $^{(1)}$. As primeiras são causadas por traumatismo direto, rápido, que aplica força compressiva sobre o tecido muscular $^{(2)}$ e, a segunda ocorre quando o alongamento causado no músculo ultrapassa sua capacidade elástica. Qualquer que seja o mecanismo de trauma há dor, que interfere com a locomoção, no desempenho de atividades esportivas e da vida diária ${ }^{(3-6)}$. A alta variabilidade destas lesões limita a realização de estudos $\operatorname{clínicos}^{(7)}$ e a determinação do tratamento mais eficaz ${ }^{(8)}$.

Com o objetivo de minimizar essas variabilidades, estudos prévios produziam lesão muscular invasivamente, por meio de compressão do músculo com fórceps em animais ${ }^{(9,10)}$.

Outros autores utilizaram modelos experimentais de lesão muscular em animais por meio de técnicas não invasivas, como sistemas de martelo com mola ${ }^{(11)}$ e técnicas de liberação de peso, de alturas predeterminadas ${ }^{(6,12-14)}$.

Em 1975, Järvinen e Sorvari ${ }^{(15)}$ desenvolveram o modelo experimental de lesão muscular utilizando martelo com mola. Os autores produziram lesão do tríceps sural e constataram, dois dias após, a uniformidade das alterações produzidas. Outros autores ${ }^{(16)}$ utilizaram a técnica de liberação de massa (700g), de uma altura de 12,5cm e constataram a importância em se determinar a força, a deformação tecidual e a energia transferida ao músculo. Em outra ocasião ${ }^{(12)}$, a técnica de liberação de massa também foi utilizada. No entanto, esses autores utilizaram altura de $102 \mathrm{~cm}$ e massa de $171 \mathrm{~g}$, acoplaram uma célula de carga ao sistema para permitir o cálculo da energia absorvida pelo tecido muscular. Apesar de estudos desta natureza, a determinação da massa e altura, ideais para realização deste tipo de lesão, ainda é discutível.

Assim como as técnicas experimentais de lesão muscular, os tratamentos delas também vari- am ${ }^{(12)}$. São empregadas técnicas de imobilização e remobilização ${ }^{(15)}$, crioterapia $^{(17)}$ e princípio Rice - rest, ice, compression, elevation, medicações anti-inflamatórias não estereoidais $^{(18)}$ ou corticoides $^{(19)}$, ultrassom terapêutico ${ }^{(11)}$, laserterapia ${ }^{(20-22)}$ e fatores de crescimento ${ }^{(23)}$ com a intenção de promover a reparação mais rápida da lesão muscular. Apesar destes esforços, o melhor tratamento da lesão muscular ainda permanece inconclusivo ${ }^{(12)}$.

Assim, o objetivo deste estudo foi avaliar, experimentalmente, o efeito da aplicação do laser terapêutico na reparação do tecido muscular, submetido à lesão por contusão, utilizando metodologias de ensaio mecânico e histológica.

\section{MATERIAIS E MÉTODOS}

O projeto desta pesquisa foi aprovado pelo Comitê de Ética em Experimentação Animal da Faculdade de Medicina de Ribeirão Preto - Cetea/FMRP (protocolo $\mathrm{n}^{\mathrm{o}}$ 069/2006).

Foram utilizadas 45 ratas adultas da linhagem Wistar, com peso corporal médio de $300 \mathrm{~g}$. Os animais foram divididos em três grupos experimentais ( $n=15$ /grupo): grupo C (controle, sem lesão), grupo LM (lesão muscular por esmagamento e mantidos sem tratamento), e grupo LM-L (lesão muscular por esmagamento e tratamento com laser AsGa. 960nm).

Para realização da lesão muscular, houve administração prévia dos anestésicos xilazina e quetamina $(0,1 \mathrm{ml} / 100 \mathrm{~g})$ e tricotomia na região posterior da perna dos animais, na região do músculo gastrocnêmio. O método utilizado para causar o esmagamento muscular foi a técnica de liberação de peso a partir de uma altura preestabelecida e padronizada, empregada em outros estudos $^{(6,12-14)}$. Os animais foram posicionados sob esfera de chumbo de $250 \mathrm{~g}$, que foi liberada a partir da altura de $30 \mathrm{~cm}$ (Figura 1).

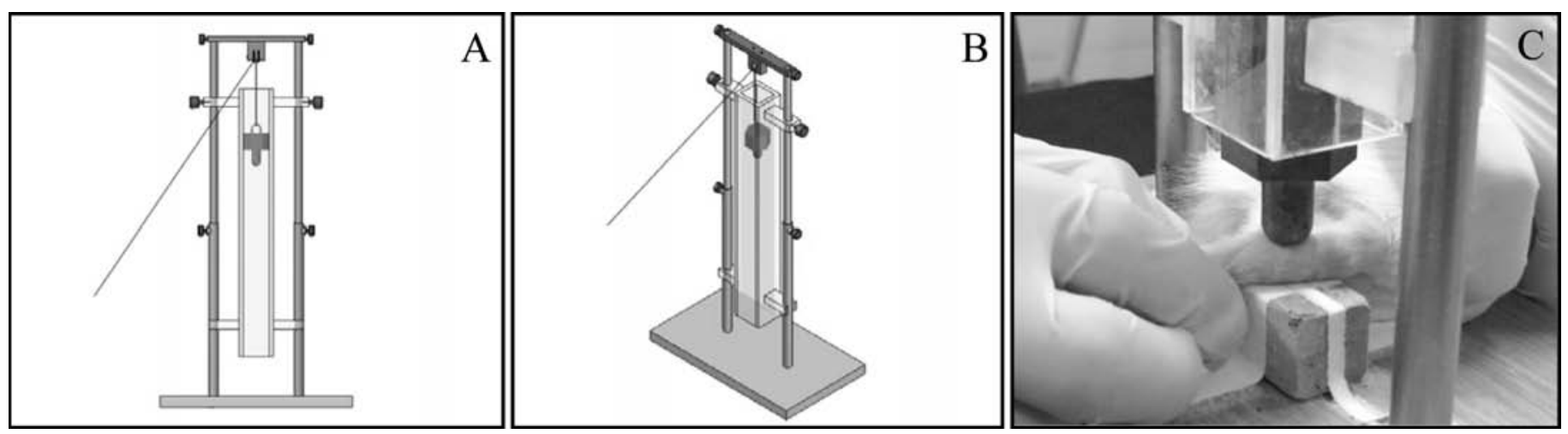

Figura 1 - Desenho esquemático do equipamento utilizado para produzir lesão experimental por esmagamento em músculo de animais de pequeno porte (A e B). Posicionamento do animal para realização da lesão muscular (C). 
Os animais do grupo LM-L foram submetidos à aplicação do laser AsGa imediatamente após a lesão muscular e, depois, duas vezes ao dia, durante três dias. Para aplicação do laser, os animais foram mantidos sob contenção manual, sem anestesia prévia Foi utilizada dose de $2 \mathrm{~J} / \mathrm{cm}^{2}$ com tempo de aplicação calculado automaticamente pelo aparelho.

Ao término do período experimental, os animais foram submetidos à eutanásia com dose excessiva de hidrato de cloral. Os músculos gastrocnêmios foram colhidos de modo a preservar a origem e a inserção e destinados às análises mecânicas ( $\mathrm{n}=10$ /grupo) e histológicas ( $n=5 /$ grupo). Os ensaios mecânicos foram realizados em máquina universal de ensaios (Emic ${ }^{\circledR}$ ), modelo DL 10000, no Laboratório de Bioengenharia da FMRP/USP. A velocidade de aplicação de carga foi de $10 \mathrm{~mm} / \mathrm{min}$, com pré-carga de $5 \mathrm{~N}$ e tempo de acomodação de 30s (Figura 2).

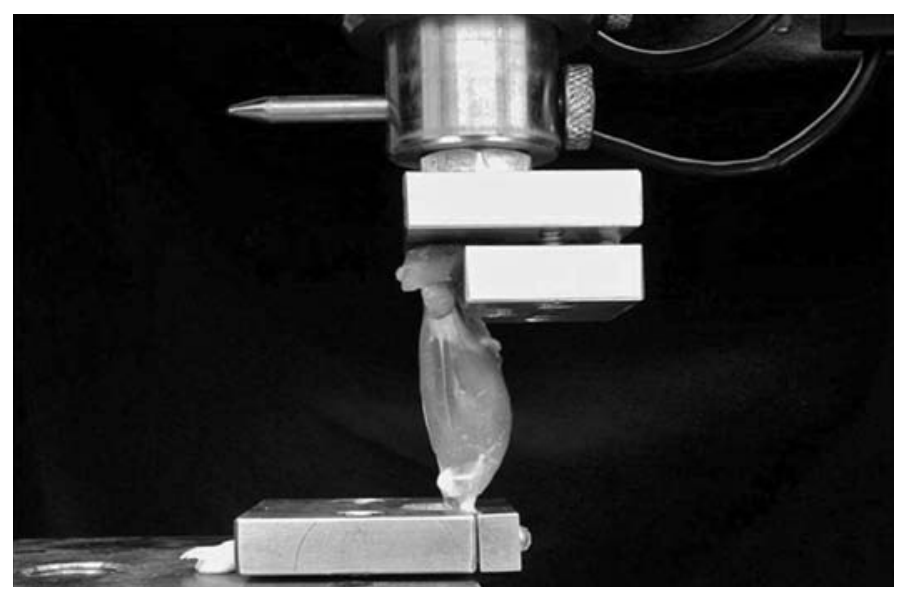

Figura 2 - Montagem e acoplamento do músculo gastrocnêmio aos acessórios da máquina universal de ensaios, pronto para a realização dos ensaios mecânicos de tração

Os parâmetros utilizados para avaliação e comparação dos dados foram a força máxima e rigidez relativa.

Para o preparo histológico, os músculos foram mantidos, após a dissecção, sete dias em recipiente com formol. Passado o período de sete dias, os músculos foram retirados do formol e submetidos a cortes transversais, com bisturi cirúrgico, na região central do ventre muscular, da face mais externa do músculo para a mais interna, formando pequenos blocos de $1 \mathrm{~cm}^{2}$.

Posteriormente, a inclusão desses blocos em uma série ascendente de alcoóis a 70, 80 e 90\% de concentração e álcool absoluto, por um período de 24 horas, foi iniciada. Em seguida, os blocos foram mergulhados em xilol e banhados três vezes em parafina derretida, para realizar o emblocamento e futuro corte no micrótomo.
Os cortes foram feitos transversalmente à fibra muscular, com uma espessura de $6 \mu \mathrm{m}$, obtidas com navalhas de vidro através de um ultramicrótomo MT 6000 - XF (RMC Inc.), formando lâminas semifinas de tecido. Após este processo, a hidratação da peça e desparafinação em uma estufa foram realizadas. Inicia-se então a montagem das lâminas que, quando prontas, foram coradas com hematoxilina-eosina (H\&E) e fixadas com resina Permot $^{\circledR}$ para posterior análise em microscopia de luz, com aumento definido, e analisador de imagens, para observação da área de hematoma e outras alterações visíveis.

As secções transversais semifinas dos segmentos foram observadas em um fotomicroscópio Axioimager M1 (Carl Zeiss) usando as objetivas 20, 40 e/ou 100 vezes, com imersão em óleo. As imagens dos músculos foram digitalizadas através de câmera de alta resolução Axiocam MR5 (Carl Zeiss) para um microcomputador (IBM/ PC - AT Pentium ${ }^{\circledR}$ III) no qual foram armazenadas no disco rígido, para posterior análise. As áreas de hematoma foram identificadas por inspeção visual e contadas.

Os resultados foram submetidos ao teste de normalidade, para verificar se o comportamento dos dados foi ou não paramétrico, através da Análise de Variância Multifatorial (Anova multifatorial). Para a comparação entre os grupos foi realizado o pós-teste de Tukey. Em todas as análises foi adotado o nível de significância de 5\% (p $\leq 0,05)$.

\section{RESULTADOS}

\section{Análise mecânica}

Foram ensaiados 30 músculos gastrocnêmios, sendo os valores expressos em médias e desvios padrão para cada uma das propriedades dos três grupos analisados.

\section{Força máxima}

As médias dos valores de força máxima foram: $(35,70 \pm 2,69 \mathrm{~N})$ no grupo $\mathrm{C},(31,77 \pm 2,59) \mathrm{N}$ no grupo LM e $(34,36 \pm 3,63) \mathrm{N}$ no grupo LM-L. Foi observada diferença estatística significativa entre os grupos $\mathrm{C}$ e LM (p < 0,05) (Figura 3).

\section{Rigidez relativa}

As médias dos valores de rigidez relativa foram: $(3,75 \pm 0,98) \mathrm{N} / \mathrm{mm}$ no grupo C, $(3,84 \pm 0,32) \mathrm{N} / \mathrm{mm}$ no grupo LM e $(4,43 \pm 0,68) \mathrm{N} / \mathrm{mm}$ no grupo LM-L. Não foi observada diferença estatística significativa entre os grupos ( $>$ 0,05) (Figura 4).

\section{Análise histológica}

No terceiro dia pós-lesão, os cortes histológicos dos músculos dos grupos LM e LM-L demonstraram a presença de hematomas, infiltrado celular e desorganização 


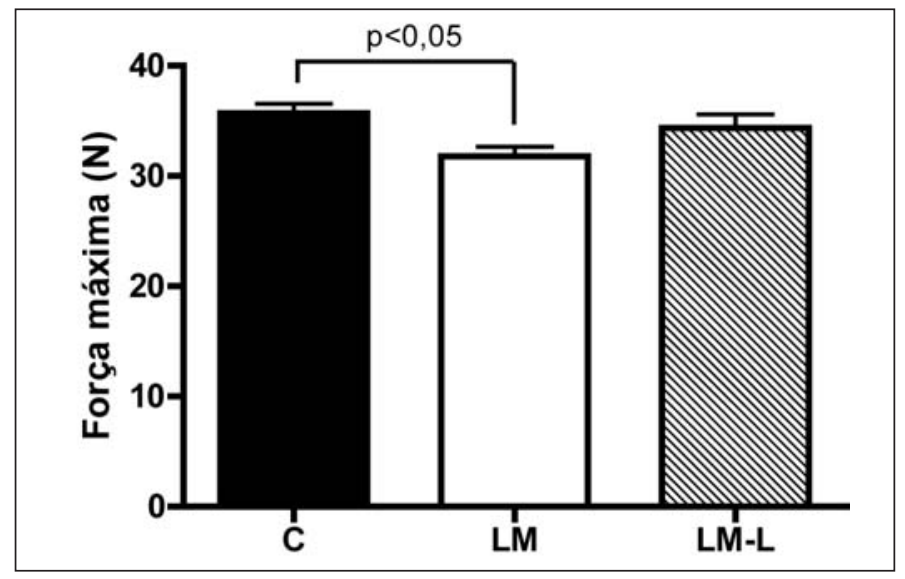

Figura 3 - Médias dos valores de força máxima

tecidual. Já o aumento de 40 vezes permitiu observar neovascularização no grupo LM-L e hematoma em processo de reparação (Figura 5).

\section{DISCUSSÃO}

As lesões musculares são uma das mais comuns formas de lesões decorrentes do esporte ${ }^{(2,19)}$ e que podem ser causadas por contusão, estiramento ou laceração ${ }^{(2)}$, com predomínio das contusões e estiramentos ${ }^{(1)}$.

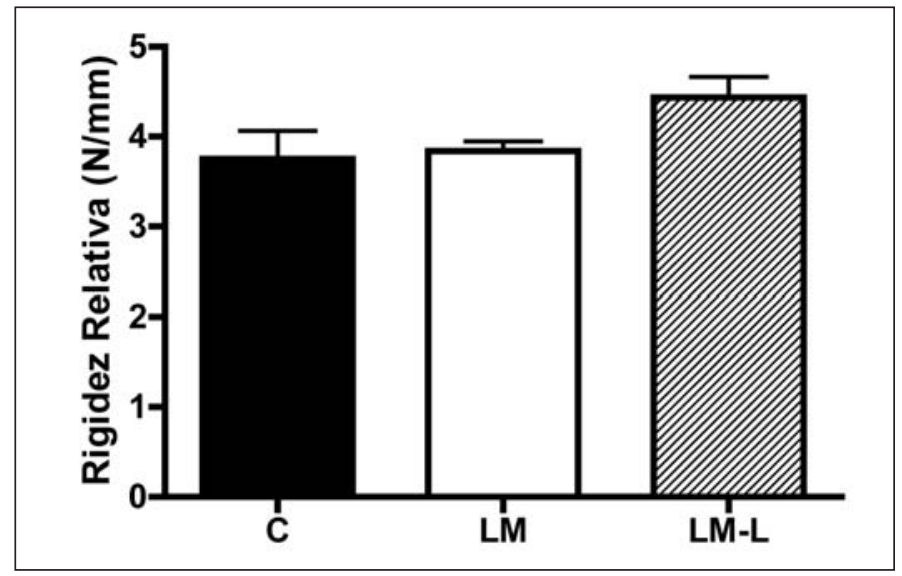

Figura 4 - Médias dos valores da rigidez relativa

Apesar da alta incidência das lesões musculares por contusão, há limitação de estudos nesta área, devido à alta variabilidade das lesões $^{(7)}$. Sendo assim, os modelos experimentais de produção padronizada de lesão muscular são de grande importância na tentativa de elucidar os métodos de reabilitação da contusão muscular. Há várias técnicas para produção de lesão muscular do tipo contusão, sendo alguns invasivos, através de um fórceps inserido pela incisão na pele ${ }^{(10)}$ e outros, não invasivos, que utilizam

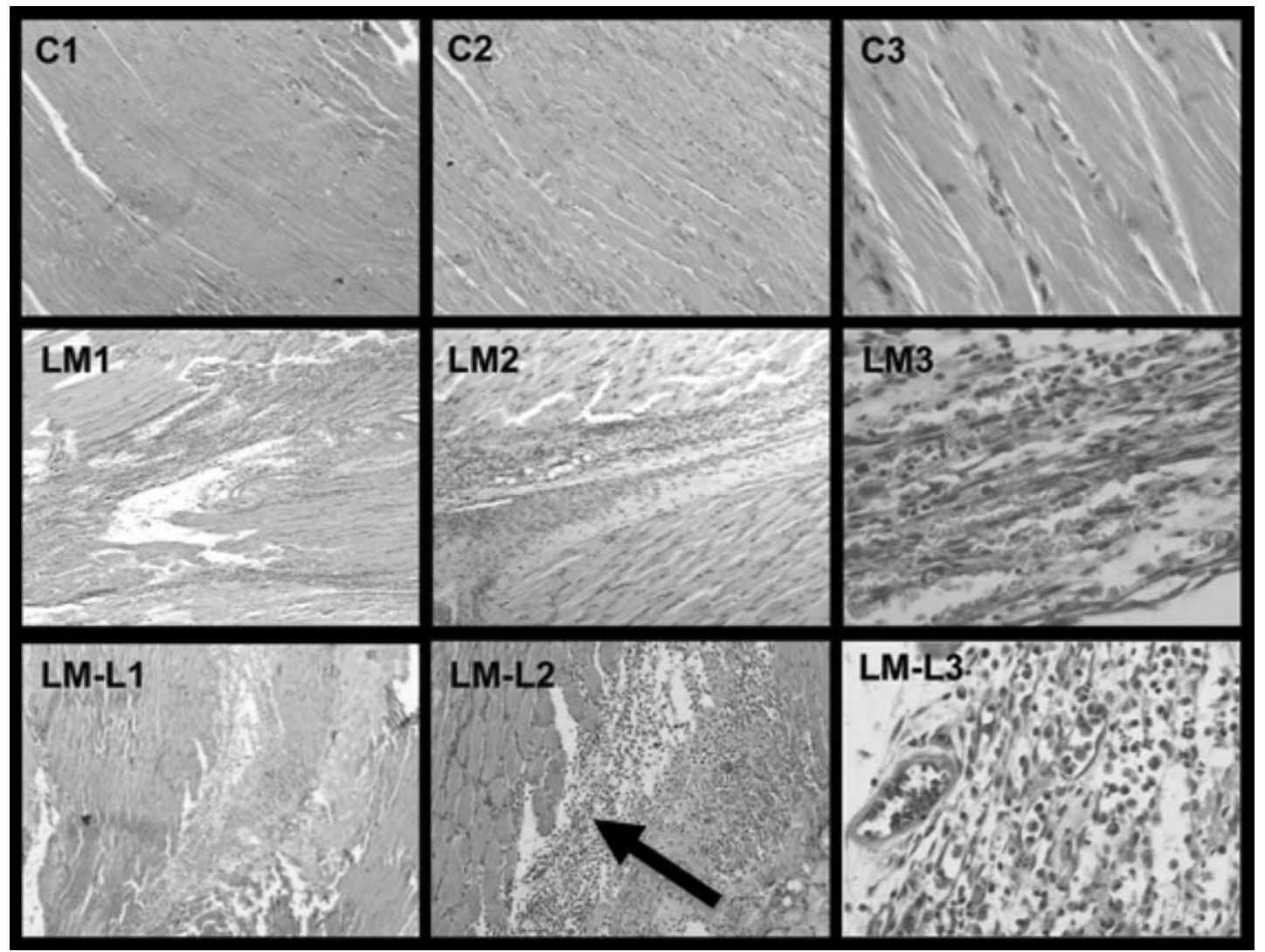

Figura 5 - Fibras musculares do músculo gastrocnêmio dos grupos controle (C), lesão muscular (LM) e lesão muscular cm laserterapia (LM-L). A seta aponta para vaso sanguíneo formado no grupo LM-L. Imagem capturada com objetivas de cinco (C1, LM1 e LM-L1), 10 (C2, LM2 e LM-L2) e 40 (C3, LM3 e LM-L3). 
sistemas de martelo com mola ${ }^{(11)}$ e técnicas de liberação de peso $^{(6,12-14,16)}$. Após estabelecida e padronizada a metodologia de lesão muscular é possível administrar e comparar técnicas e procedimentos para acelerar a recuperação do músculo. As técnicas são variadas, incluindo agentes físicos, como o gelo ${ }^{(24)}$; agentes medicamentosos ${ }^{(8)}$; agentes mecânicos, como a imobilização ${ }^{(25)}$; e recursos da eletroterapia, como o ultrassom de baixa potência ${ }^{(11)}$ e laser $^{(20-22)}$. Entretanto, a melhor técnica não está totalmente elucidada e há divergência de resultados na literatura.

Oliveira et $a^{(20)}$ estudaram o efeito do laser AsGa na regeneração do músculo tibial anterior de ratos e submetidos a tratamento por cinco dias consecutivos, nas respectivas doses, $3 \mathrm{~J} / \mathrm{cm}^{2}$ e $10 \mathrm{~J} / \mathrm{cm}^{2}$. Os autores concluíram que os grupos tratados apresentaram aspectos morfológicos semelhantes aos do grupo controle, mas foi observado aumento da massa muscular no grupo irradiado com $10 \mathrm{~J} / \mathrm{cm}^{2}$.

Em outro estudo mais recente ${ }^{(22)}$, os pesquisadores observaram que a aplicação do laser AsGa durante sete dias, com doses de $5 \mathrm{~J} / \mathrm{cm}^{2}$ e $10 \mathrm{~J} / \mathrm{cm}^{2}$, proporcionou aumento da atividade mitocondrial, da ação dos fibroblastos e macrófagos e a angiogênese do músculo tibial anterior de roedores.

Em nosso estudo foi observada diferença da força máxima entre o grupo controle e o grupo de animais submetidos à lesão muscular e mantidos sem tratamento, constatando a perda da resistência mecânica do músculo

\section{REFERÊNCIAS}

1. Armfield DR, Kim DH, Towers JD, Bradley JP, Robertson DD. Sports-related muscle injury in the lower extremity. Clin Sports Med. 2006;25(4):803-42.

2. Garrett WE Jr. Muscle strain injuries. Am J Sports Med. 1996;24(6 Suppl):S2-8.

3. Arrington ED, Miller MD. Skeletal muscle injuries. Orthop Clin North Am. 1995;26(3):411-22.

4. Järvinen TA, Järvinen $T L$, Kääriäinen $M$, Aärimaa $V$, Vaittinen $S$, Kalimo $H$, et al. Muscle injuries: optimising recovery. Best Pract Res Clin Rheumatol. 2007;21(2):317-31.

5. Minamoto VB, Grazziano CR, Salvini TF. Effect of single and periodic contusion on the rat soleus muscle at different stages of regeneration. Anat Rec. 1999;254(2):281-7.

6. Minamoto VB, Bunho SR, Salvini TF. Regenerated rat skeletal muscle after periodic contusions. Braz J Med Biol Res. 2001;34(11):1447-52

7. Aspelin $\mathrm{P}$, Ekberg $\mathrm{O}$, Thorsson $\mathrm{O}$, Wilhelmsson $\mathrm{M}$, Westlin $\mathrm{N}$. Ultrasound examination of soft tissue injury of the lower limb in athletes. Am J Sports Med.1992;20(5):601-3.

8. Beiner JM, Jokl P. Muscle contusion injury and myositis ossificans traumatica. Clin Orthop Relat Res. 2002;(403 Suppl):S110-9.

9. Allbrook D. An electron microscopic study of regenerating skeletal muscle. J Anat. 1962;96:137-52.

10. Bunn JR, Canning J, Burke G, Mushipe M, Marsh DR, Li G. Production of consistent crush lesions in murine quadriceps muscle--a biomechanical, histomorphological and immunohistochemical study. J Orthop Res. 2004;22(6):1336-44.

11. Rantanen J, Thorsson O, Wollmer P, Hurme T, Kalimo H. Effects of therapeutic ultrasound on the regeneration of skeletal myofibers after experimental muscle injury. Am J Sports Med. 1999;27(1):54-9.

12. Crisco JJ, Jokl P, Heinen GT, Connell MD, Panjabi MM. A muscle contusion injury model. Biomechanics, physiology, and histology. Am J Sports Med. 1994;22(5):702-10.

13. Fisher BD, Baracos VE, Shnitka TK, Mendryk SW, Reid DC. Ultrastructural events following acute muscle trauma. Med Sci Sports Exerc. 1990;22(2):185-93.

14. Wright-Carpenter T, Opolon P, Appell HJ, Meijer H, Wehling P, Mir LM. Treatment of muscle injuries by local administration of autologous conditioned serum: animal ex- lesado. Na comparação entre o grupo controle e o grupo de animais submetidos à lesão muscular e laserterapia não foi observada diferença, ou seja, a aplicação do laser promoveu a recuperação mecânica do músculo previamente lesado. Com relação à rigidez, não foi observada diferença estatística entre os grupos, mas o grupo de animais submetidos ao tratamento com laser apresentou maior rigidez que os demais grupos.

A análise histológica foi realizada nos três grupos de três dias (C, LM e LM-L), para fins de comparação.

Foi observada diferença morfológica entre os músculos dos ratos submetidos à lesão muscular. Os achados histológicos permitiram observar hematomas evidentes nos grupos submetidos à lesão muscular (LM e LM-L), vale ressaltar que o grupo de animais tratados com laser (LM-L) apresentou início de reparação tecidual, com menor desorganização celular e área de lesão. Além disso, foi possível observar formação de vasos neste grupo, constatando a capacidade de angiogênese da laserterapia. Não foi realizada análise estatística dos resultados histológicos, pois não foi nosso objetivo avaliar quantitativamente as lâminas.

\section{CONCLUSÃO}

Os resultados deste estudo permitem concluir que a laserterapia influencia de forma positiva no processo de regeneração da lesão muscular. periments using a muscle contusion model. Int J Sports Med. 2004;25(8):582-7.

15. Järvinen M, Sorvari T. Healing of a crush injury in rat striated muscle. 1. Description and testing of a new method of inducing a standard injury to the calf muscles. Acta Pathol Microbiol Scand A. 1975;83(2):259-65.

16. Francis RS, Heckmann RA, Stratton SA. Therapeutic ultrasound - Its effect on the healing time of contusions. Med Sci Sports Exerc.1983;15(2):154.

17. Schaser KD, Disch AC, Stover JF, Lauffer A, Bail HJ, Mittlmeier T. Prolonged superficial local cryotherapy attenuates microcirculatory impairment, regional inflammation, and muscle necrosis after closed soft tissue injury in rats. Am J Sports Med. 2007;35(1):93-102.

18. Rahusen FT, Weinhold PS, Almekinders LC. Nonsteroidal anti-inflammatory drugs and acetaminophen in the treatment of an acute muscle injury. Am J Sports Med. 2004;32(8):1856-9.

19. Beiner JM, Jokl P, Cholewicki J, Panjabi MM. The effect of anabolic steroids and corticosteroids on healing of muscle contusion injury. Am J Sports Med. 1999;27(1):2-9

20. Oliveira NM, Parizzotto NA, Salvini TF. GaAs (904-nm) laser radiation does not affect muscle regeneration in mouse skeletal muscle. Lasers Surg Med. 1999;25(1):13-21.

21. Amaral AC, Parizotto NA, Salvini TF. Dose-dependency of low-energy HeNe laser effect in regeneration of skeletal muscle in mice. Lasers Med Sci. 2001;16(1):44-51.

22. Iyomasa DM, Garavelo I, Iyomasa MM, Watanabe IS, Issa JP. Ultrastructural analysis of the low level laser therapy effects on the lesioned anterior tibial muscle in the gerbil. Micron. 2009;40(4):413-8.

23. Takahashi T, Ishida K, Itoh K, Konishi Y, Yagyu KI, Tominaga A, et al. IGF-I gene transfer by electroporation promotes regeneration in a muscle injury model. Gene Ther. 2003;10(8):612-20.

24. Lee $\mathrm{H}$, Natsui $\mathrm{H}$, Akimoto $\mathrm{T}$, Yanagi $\mathrm{K}$, Ohshima N, Kono I. Effects of cryotherapy after contusion using real-time intravital microscopy. Med Sci Sports Exerc. 2005;37(7):1093-8.

25. Aronen JG, Garrick JG, Chronister RD, McDevitt ER. Quadriceps contusions: clinical results of immediate immobilization in 120 degrees of knee flexion. Clin J Sport Med. 2006;16(5):383-7. 